\title{
Pulse-Triggered DTI Sequence with Reduced FOV and Coronal Acquisition at 3T for the Assessment of the Cervical Spinal Cord in Patients with Myelitis
}

\author{
J. Hodel, P. Besson, O. Outteryck, H. Zéphir, D. Ducreux, A. Monnet, D. Chéchin, M. Zins,
} M. Rodallec, J.P. Pruvo, P. Vermersch, and X. Leclerc

\begin{abstract}
BACKGROUND AND PURPOSE: DTI is a promising technique for imaging of the spinal cord, but the technique has susceptibility-induced artifacts. We evaluated a pulse-triggered DTI sequence with an rFOV technique and coronal acquisition for the assessment of the cervical spinal cord in patients with myelitis at 3T.
\end{abstract}

MATERIALS AND METHODS: A rFOV acquisition was established by a noncoplanar application of the excitation and the refocusing pulse in conjunction with outer volume suppression. The DTI sequence was performed in the coronal plane in 12 healthy volunteers and 40 consecutive patients with myelitis. Probabilistic tractography of the posterior and lateral funiculi was performed from the $\mathrm{Cl}$ to $\mathrm{C} 7$ levels. $\mathrm{FA}, \mathrm{MD}, \mathrm{aD}, \mathrm{rD}$, and ratios of $\mathrm{aD}$ and $\mathrm{rD}$ were measured.

RESULTS: In healthy volunteers, mean DTI indices within the whole-fiber pathways were the following: $F A=0.61, \mathrm{MD}^{2}=1.17 \times 10^{-3} \mathrm{~mm} / \mathrm{s}$, $\mathrm{aD}=1.96 \times 10^{-3} \mathrm{~mm}^{2} / \mathrm{s}, \mathrm{rD}=0.77 \times 10^{-3} \mathrm{~mm}^{2} / \mathrm{s}$, and ratios of $\mathrm{aD}$ and $\mathrm{rD}=2.5$. Comparison of healthy controls and patients with myelitis identified statistically significant differences for all DTI parameters. Different patterns of myelitis, including spinal cord atrophy and active inflammatory lesions, were recognized. There was a significant correlation between clinical severity and DTI parameters.

CONCLUSIONS: The present work introduces a new approach for DTI of the cervical spinal cord at 3T, enabling a quantitative follow-up of patients with myelitis.

ABBREVIATIONS: $\mathrm{aD}=$ axial diffusivity; $\mathrm{FA}=$ fractional anisotropy; $\mathrm{HC}=$ healthy controls; $\mathrm{MD}=$ mean diffusivity; $\mathrm{rD}=$ radial diffusivity; $\mathrm{rFOV}=$ reduced FOV

S everal inflammatory diseases, such as MS, neuromyelitis optica, or autoimmune diseases, may involve the spinal cord. Clinical disability is partly dependent on spinal cord lesions. Several studies reported the value of DTI for imaging the spinal cord at $1.5 \mathrm{~T},{ }^{1-5}$ particularly for the diagnosis of myelitis. ${ }^{6-9}$ DTI parameters such as $\mathrm{FA}, \mathrm{MD}, \mathrm{aD}$, and $\mathrm{rD}$ may help to distinguish myelin repair from axonal loss ${ }^{10}$ or improve the detection of occult spinal cord pathology. ${ }^{6,8,11}$ However, a quantitative and reliable DTI is required for patients with myelitis scanned at 3T.

Most of the previous studies used single-shot EPI to achieve DTI due to its high signal-to-noise ratio and its insensitivity to motion-

Received March 27, 2012; accepted after revision June 12.

From the Departments of Neuroradiology (J.H., P.B., A.M., J.P.P., X.L.) and Neurology (O.O., H.Z., P.V.), CHU Salengro, Lille, France; Department of Radiology (J.H., M.Z.,

M.R.), Hôpital Saint Joseph, Paris, France; Department of Neuroradiology (D.D.),

CHU Bicêtre, Le Kremlin-Bicêtre, France; and Philips Medical Systems (D.C.), Suresnes, France.

Please address correspondence to Jérôme Hodel, MD, Department of Radiology, Hôpital Saint Joseph, 185 Rue Raymond Losserand, Paris 75014, France; e-mail: jeromehodel@hotmail.com

2 EBMidence-Based Medicine Level 2

http://dx.doi.org/10.3174/ajnr.A3254 related artifacts. However, DTI of the spinal cord remains challenging. ${ }^{12,13}$ Indeed, single-shot EPI is prone to distortions due to B0 inhomogeneities, particularly at 3T. Distortions are related to a long echo-planar readout train leading to phase-error accumulation. In this study, we evaluated a rFOV technique, which includes both outer volume suppression and inner volume excitation. ${ }^{14,15}$ Such a technique is particularly suitable for the imaging of patients with myelitis because the spinal cord size allows ample truncation of the FOV in at least 1 dimension. Reducing the FOV in the phase-encoding direction leads to a drastic shortening of the EPI readout with reduced susceptibility-related artifacts.

No study has yet evaluated rFOV DTI in patients with MS at 3T, to our knowledge. The aim of this study was to evaluate a pulsetriggered DTI sequence acquired in the coronal plane with a rFOV technique for the tractography-based measurements of diffusion indices over the cervical spinal cord in patients with myelitis at 3T.

\section{MATERIALS AND METHODS \\ Patients and Volunteers}

Twelve volunteers participated in this study, which was approved by the local ethics committee review board. From April to Sep- 
tember 2011, we recruited 40 patients with inflammatory diseases potentially affecting the spinal cord. Twenty-five patients presented with MS (15 relapsing-remitting, 5 secondary-progressive, and 5 primary progressive forms), and 15 patients presented with another cause of inflammatory myelopathy (11 neuromyelitis optica, 1 sarcoidosis, 1 Gougerot-Sjögren syndrome, 2 idiopathic). All patients underwent a complete clinical evaluation at the time of MR imaging. For each patient, 1 neurologist (O.O.) established an Expanded Disability Status Scale score (varying from 0 to 10$)^{16}$ and reported a Pyramidal Functional Score of $0-6$ and a Sensory Functional Score of $0-6$, mainly affected by spinal cord involvement. Six patients presented with a clinical relapse at the time of the study.

\section{MR Imaging}

Imaging was performed with the patient in the supine position on a 3T scanner (Achieva; Philips Medical Systems, Best, the Netherlands). A head and neck neurovascular coil (phased array coil with 16 elements) was used to scan the spinal cord of each subject. Acquisitions of healthy volunteers and patients were interleaved and obtained on the same dates.

Before starting the present study, we first optimized the DTI sequence. We tested several parameters including gating, b-value, FOV, sensitivity encoding imaging, and acquisition plane. We changed each parameter separately and assessed the image quality. In agreement with a previous study, ${ }^{17}$ we found significant distortions when using the standard DTI sequence compared with rFOV DTI, but we did not attempt to compare both techniques due to the long scanning time.

Indeed in this study, the DTI sequence was performed with a reduced-FOV technique. This technique, previously described, ${ }^{14}$ is a single-shot EPI that uses a noncoplanar application of the excitation and the refocusing pulses. By adjusting the tilt angle, this approach allows FOV reduction (and then EPI readout train) and multiple-section imaging in a time-effective way. ${ }^{14}$ This method is combined with outer volume suppression, which is performed by using a spatially selective pulse applied before the section excitation. Outer volume suppression suppresses signals coming from outside the reduced FOV to ensure that the acquisition is performed only in this reduced FOV. ${ }^{14,15}$ In this study, the DTI sequence was performed in the coronal plane with the phase-encoding direction oriented right to left to limit susceptibility artifacts related to bones and lungs. We obtained a reduced FOV in the phase-encoding direction with the following FOV dimensions: right $/$ left $=42 \mathrm{~mm}$ and feet $/$ head $=170 \mathrm{~mm}$, leading to a reduced EPI readout train with 35 phase-encoding lines. The acquired matrix was $96 \times 23$, leading to a voxel size of $1.77 \times$ $1.84 \times 2.5 \mathrm{~mm}$, whereas the reconstructed matrix was 160 , leading to a reconstructed voxel size of $1.06 \times 1.06 \times 2.5 \mathrm{~mm}$. The image acquisition parameters were as follows: 15 directions with $b=600, \mathrm{TE}=38.7 \mathrm{~ms}$, section thickness $=2.5 \mathrm{~mm}$, partial Fourier acquisition with half-scan factor $=0.6$, number of signal averages $=3$, flip angle $=90^{\circ}$, flow compensation. Pulse triggering was done by using a finger sensor with TR beats $=3$ (mean TR $=$ $3000 \mathrm{~ms}$ ); each section group was triggered to reduce motion artifacts. A frequency-selective fat saturation was used to suppress the fat signal (spectral presaturation with inversion recovery). The whole spinal cord was scanned in all cases by using 11 contiguous sections without spacing in the coronal plane. To improve magnetic field homogeneity, we performed a localized MR shimming on the cervical spinal cord. We did not use sensitivity encoding imaging associated with the rFOV technique to avoid inhomogeneous noise amplification due to the g-factor penalty.

The mean acquisition time of the optimized DTI sequence was 7 minutes 30 seconds according to the heart rate. There were no changes in the MR imaging hardware/software during the entire study (52 MR imaging examinations).

Sagittal (turbo spin-echo, $\mathrm{TE}=80 \mathrm{~ms}$; TR $=3465 \mathrm{~ms}$; section thickness $=3 \mathrm{~mm}$; frequency-selective fat saturation spectralattenuated inversion recovery) and axial (fast-field echo, $\mathrm{TE}=6.9$ $\mathrm{ms}$; TR $=683 \mathrm{~ms}$; section thickness $=3.5 \mathrm{~mm}$ ) T2-weighted acquisitions of the spinal cord were obtained in all subjects. In addition, sagittal T1-weighted acquisitions (turbo spin-echo, $\mathrm{TE}=7.5 \mathrm{~ms}$; $\mathrm{TR}=530 \mathrm{~ms}$; section thickness $=3 \mathrm{~mm}$ ) were obtained in patients with and without gadolinium enhancement.

The total acquisition lasted 17 minutes in volunteers and 25 minutes in patients.

\section{Image Analysis}

T1- and T2-Weighted Imaging. One neuroradiologist (J.H.) analyzed all images. On the basis of T1- and T2-weighted images, the reader assessed the occurrence of spinal cord T2 hyperintensities, spinal cord atrophy, and gadolinium enhancement.

DTI Image Quality. Image quality was qualitatively evaluated in consensus by 2 readers (M.R. and X.L.) for both healthy volunteers and patients. The occurrence of abnormal fiber-bundle deviation or interruption related to susceptibility artifacts was analyzed by viewing the tracts registered on T2-weighted acquisitions in $3 \mathrm{D}$ mode.

Tractography. Motion-related misalignment of the diffusion tensor MR images was corrected by using the available Automated Image Registration software. Data were converted to the NIfTI format by using the Dcm2nii software (http://www.mccaus landcenter.sc.edu/mricro/mricron/dcm2nii.html). One neuroradiologist (J.H.) drew ROIs as seed masks and termination masks by using the MD images in the axial plane at the $\mathrm{C} 1$ and $\mathrm{C} 7$ levels. ROIs are described in Fig 1. Axial T2-weighted images were registered on MD images to improve the delineation of ROIs. There was a consistent sparing of the outer margin of the cervical cord to minimize volume averaging with the CSF. Probabilistic tractography was performed for each subject by using FMRIB Diffusion Toolbox software (http://www.fmrib.ox.ac.uk/fsl/ fdt/index.html) ${ }^{18,19}$ Due to the termination masks, the pathways did not extend beyond the $\mathrm{C} 1$ or $\mathrm{C} 7$ levels. One tract including posterior and lateral cord funiculi was then obtained for each subject.

DTI Index Measurement within the Tracts. For all patients and healthy volunteers, FA, MD, and eigenvalues (E1, E2, and E3) were calculated within the fiber tracts. $\mathrm{aD}$ was defined as the largest eigenvalue, and $\mathrm{rD}$ was defined as average of the second and third largest eigenvalues. We also calculated the ratio of $\mathrm{aD}$ and $\mathrm{rD}$.

AJNR Am J Neuroradiol 34:676-82 Mar 2013 www.ajnr.org 


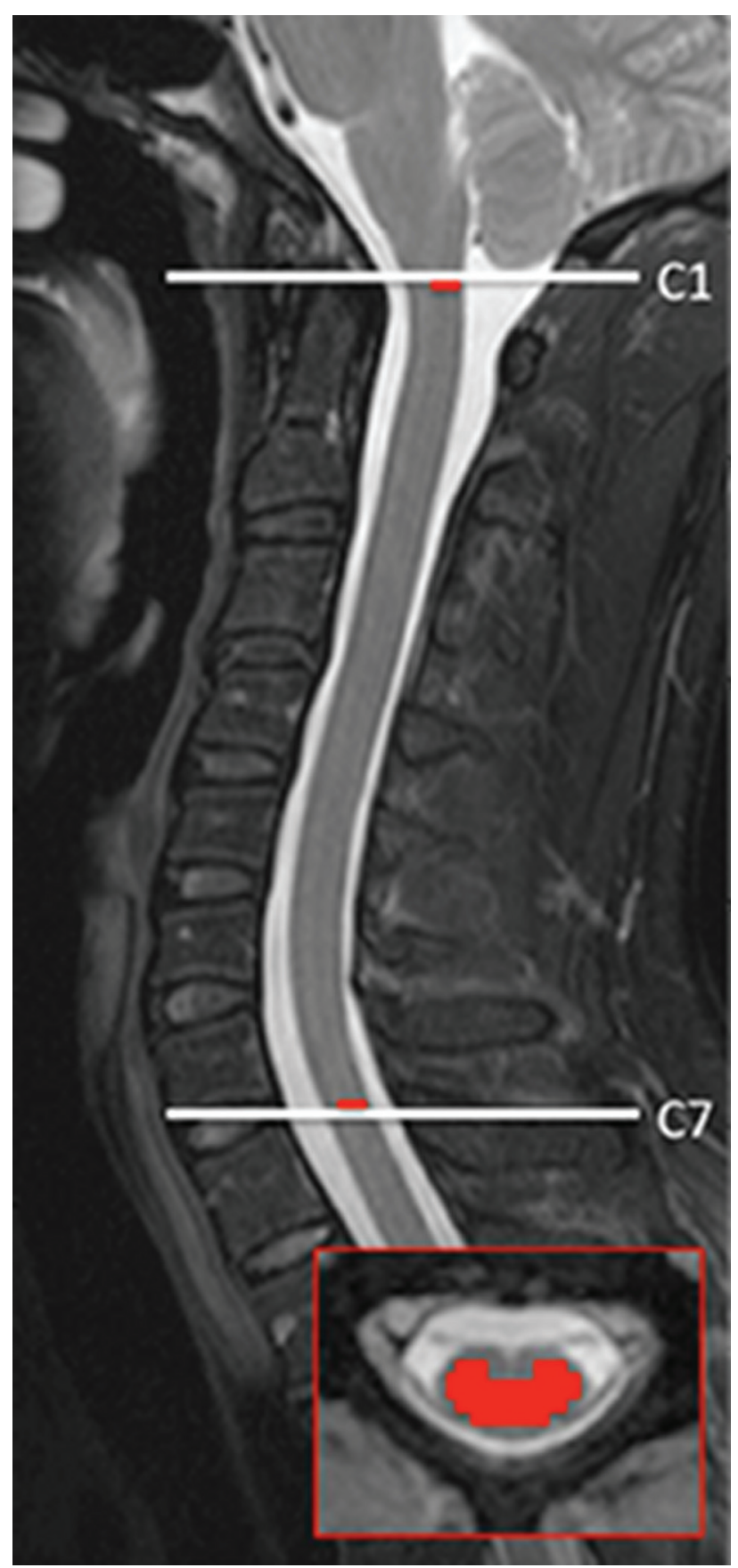

FIG 1. Midsagittal and axial T2-weighted views of a healthy volunteer (volunteer 1). Termination masks (white lines) and seed masks (red lines) were drawn at $\mathrm{Cl}$ and $\mathrm{C} 7$ levels. Due to the termination masks, the pathways did not extend beyond the $\mathrm{Cl}$ or $\mathrm{C} 7$ levels. When drawing seed masks, there was a consistent sparing of the outer margin of the cervical cord to minimize volume averaging with the CSF.

\section{Statistical Analysis}

Statistical analysis was performed (O.O.) with the SPSS software, Version 13.0 (SPSS, Chicago, Illinois). Normality of variable distribution was tested by the Shapiro-Wilk test. If a variable was normally distributed, we applied the Student $t$ test to compare means and the Pearson correlation test to measure the strength of association between different parameters. If a variable was not normally distributed, we applied the corresponding nonparametric tests: Mann-Whitney $U$ test and Spearman correlation test. To control for multiple comparisons, we applied a Bonferroni correction.

\section{RESULTS}

\section{Image Quality}

In all subjects, the fiber pathways matched their expected anatomic location on registered T2-weighted images. There was no abnormal fiber-bundle interruption reported. DTI parametric maps and tractography of a healthy volunteer are shown in Fig 2. DTI parametric maps of a patient with active lesions are shown in Fig 3.

\section{Clinical Data and Conventional Imaging}

Twelve HC and 40 patients were recruited. Cervical spinal cord MR imaging did not show any cervical lesions in 2 patients with MS and 3 patients with neuromyelitis optica. These 2 patients with MS did not present with any clinical signs of spinal cord involvement, but among the 3 patients with neuromyelitis optica, 1 had already presented with extended cervical myelitis and the 2 others had already presented with extended thoracic myelitis. All the other patients presented with at least 1 cervical lesion. Among the total cohort, 6 patients presented with a clinical relapse at the time of MR imaging, and cervical spinal cord MR imaging showed at least 1 gadolinium-enhanced lesion (5 with MS and 1 with myelitis).

\section{DTI Values and Statistical Analysis}

Mean DTI values are shown in Table 1 for patients and healthy volunteers. Comparison of HC with the total cohort and all subgroups identified statistically significant differences for almost all DTI parameters as shown in Table 2. In active inflammatory lesions, FA increased and MD decreased significantly compared with $\mathrm{HC}$ values. In the absence of active lesions and regardless of the pathology (MS or other), FA decreased and MD increased significantly.

Because the Expanded Disability Status Scale score depends on other clinical parameters such as cognitive, visual, and cerebellar impairment, we only correlated Pyramidal Functional Score and Sensory Functional Score with 2 relevant DTI parameters, FA and MD. No significant correlation was noted between age and DTI parameters in $\mathrm{HC}$ and in the whole cohort and subgroups of the cohort. In the total cohort $(n=40)$, we did not observe any significant correlation between DTI parameters and clinical parameters.

Considering all patients (MS or other) without active lesions $(n=34)$, we observed significant correlation between Sensory Functional Score and FA $(r=-0.419, P=.014 ; P=.056$ after Bonferroni correction). In the cohort of patients with MS without active lesions $(n=20)$, we observed significant correlation between Pyramidal Functional Score and FA $(r=-0.487, P=.03$; $P=.12$ after Bonferroni correction). In the cohort of patients with other causes of myelitis $(n=14)$, we observed significant correlation between Pyramidal Functional Score and MD $(r=$ -0.694, $P=.006 ; P=.024$ after Bonferroni correction). In the cohort of patients with active lesions, we did not observe any significant correlation between clinical parameters and DTI parameters.

In the cohort of patients with MS without active lesions, we 

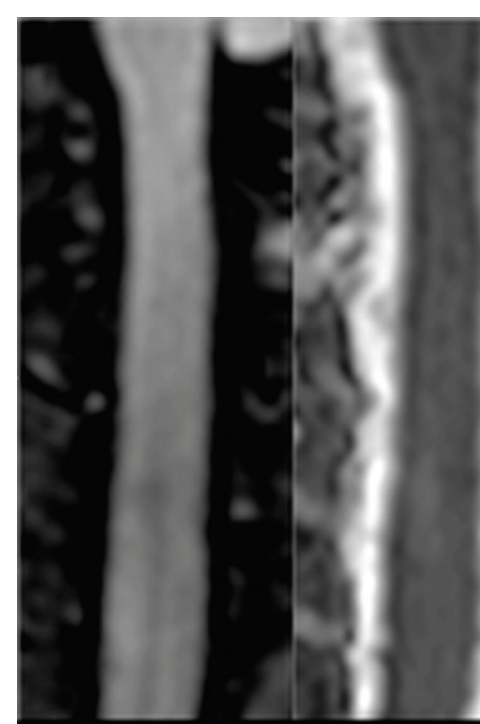

A TRACE

MD

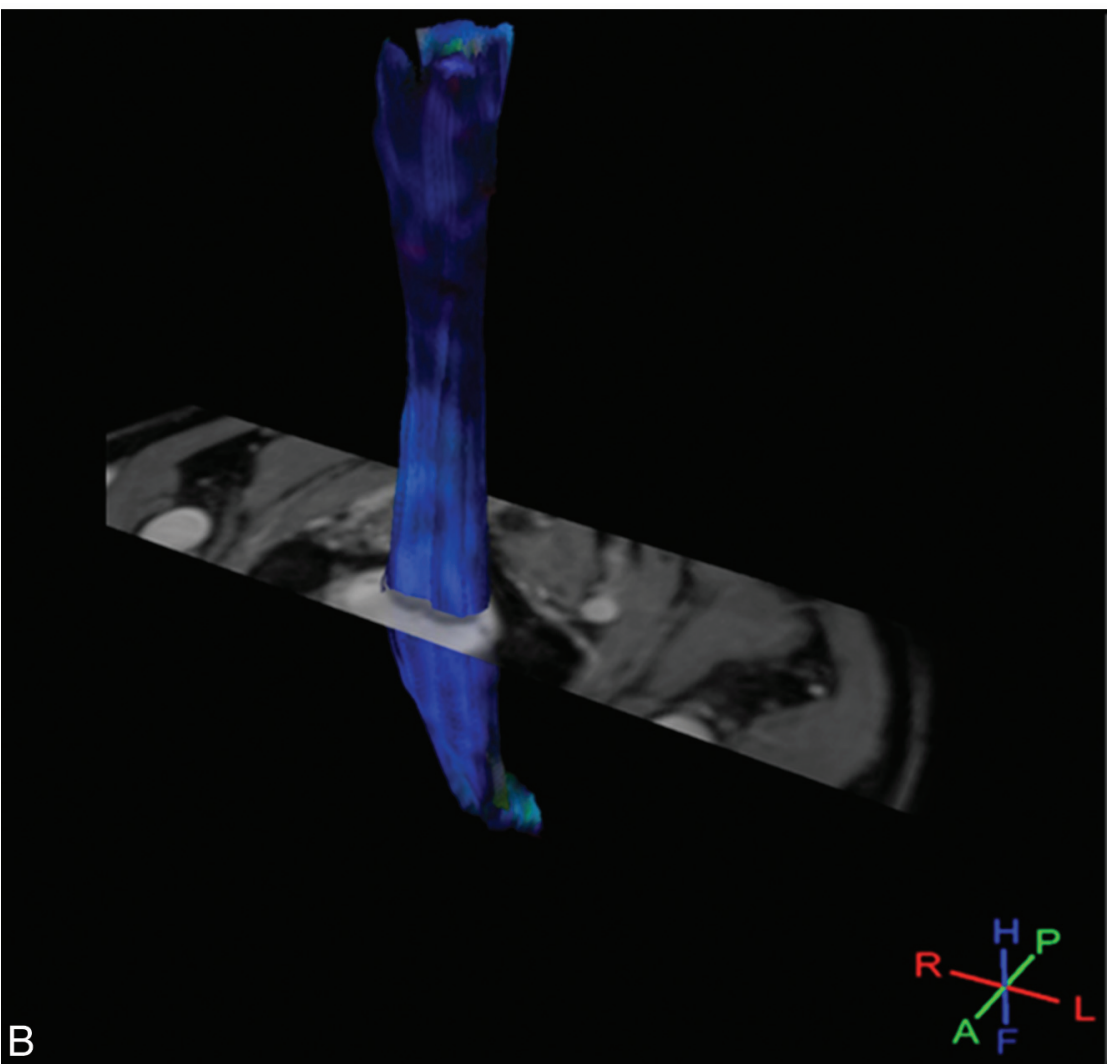

FIG 2. A, Diffusion tensor images of a healthy volunteer (volunteer 1). Parametric maps obtained in the coronal plane. Note the absence of distortion by using the rFOV DTI sequence. Longitudinal dark bands on FA maps (arrows) are related to the central gray matter in this midcoronal view of the spinal cord. B, Diffusion tensor images of a healthy volunteer (volunteer 1). 3D view of fibers obtained after probabilistic tractography displayed in a directional mode and registered on the T2-weighted image. The tracked fibers match their expected anatomic location, and no abnormal fiber bundle interruption was observed.

observed that progressive forms of MS (secondary-progressive MS, primary-progressive MS, or both) had more minor FA and ratio values than in those with relapsing-remitting MS. We also observed that patients with progressive MS had higher MD values and greater disability than those with relapsing-remitting MS (Table 3). Mean FA and ratio values were significantly $(P=.01$ and $P=.047$, respectively) lower in progressive MS $(n=8)$ than in relapsing-remitting MS $(n=$ 12). Mean MD values of progressive MS were not significantly higher than those in relapsing-remitting MS $(P=.286)$.

\section{DISCUSSION}

In this study, we aimed to quantitatively assess the cervical cord damage in patients with myelitis by using DTI at 3T. Image blurring and susceptibility-related distortions are known limitations of single-shot EPI DTI, mainly related to its long readout time in the phase-encoding direction. Reduced FOV techniques allow the reduction of susceptibility artifacts. ${ }^{20}$ In this study, we used a rFOV technique that included both inner volume excitation and outer volume suppression. Reducing the FOV in the phase-encoding direction was particularly suitable for imaging of the spinal cord and led to a drastic shortening of the EPI readout train length. In our study, the EPI readout train was shortened to 35 phase-encoding lines with a TE of $38.7 \mathrm{~ms}$, explaining reduced distortions. With the aim of reducing motion-related artifacts, we also performed a prospective gating. ${ }^{21,22}$

Region-of-interest measurements are commonly achieved for the quantitative assessment of the spinal cord with DTI, in healthy volunteers ${ }^{2}$ and in patients with $\mathrm{MS}^{9}$ or spondylotic myelopathy. ${ }^{23}$ In our study, we performed tractography-based measurements of the DTI indices. Partial volume effects may be minimized with such an approach compared with the possible inclusion of highly isotropic CSF in the ROIs. The intra- and interobserver agreement of both approaches was previously evaluated ${ }^{24}$; they were low with ROIs and excellent with tractography. We used the FMRIB Diffusion Toolbox ${ }^{18,19}$ to perform probabilistic tractography. In comparison with a tracking algorithm based on FA values, this approach may be more accurate considering the changes in terms of FA values observed in inflammatory lesions. The quantitative diffusion measures that we found are within the range of the previously reported values. ${ }^{7,24-30}$ Particularly, our results are in agreement with previous studies that used tractography-based measurements of the DTI indices. ${ }^{24,30}$ 


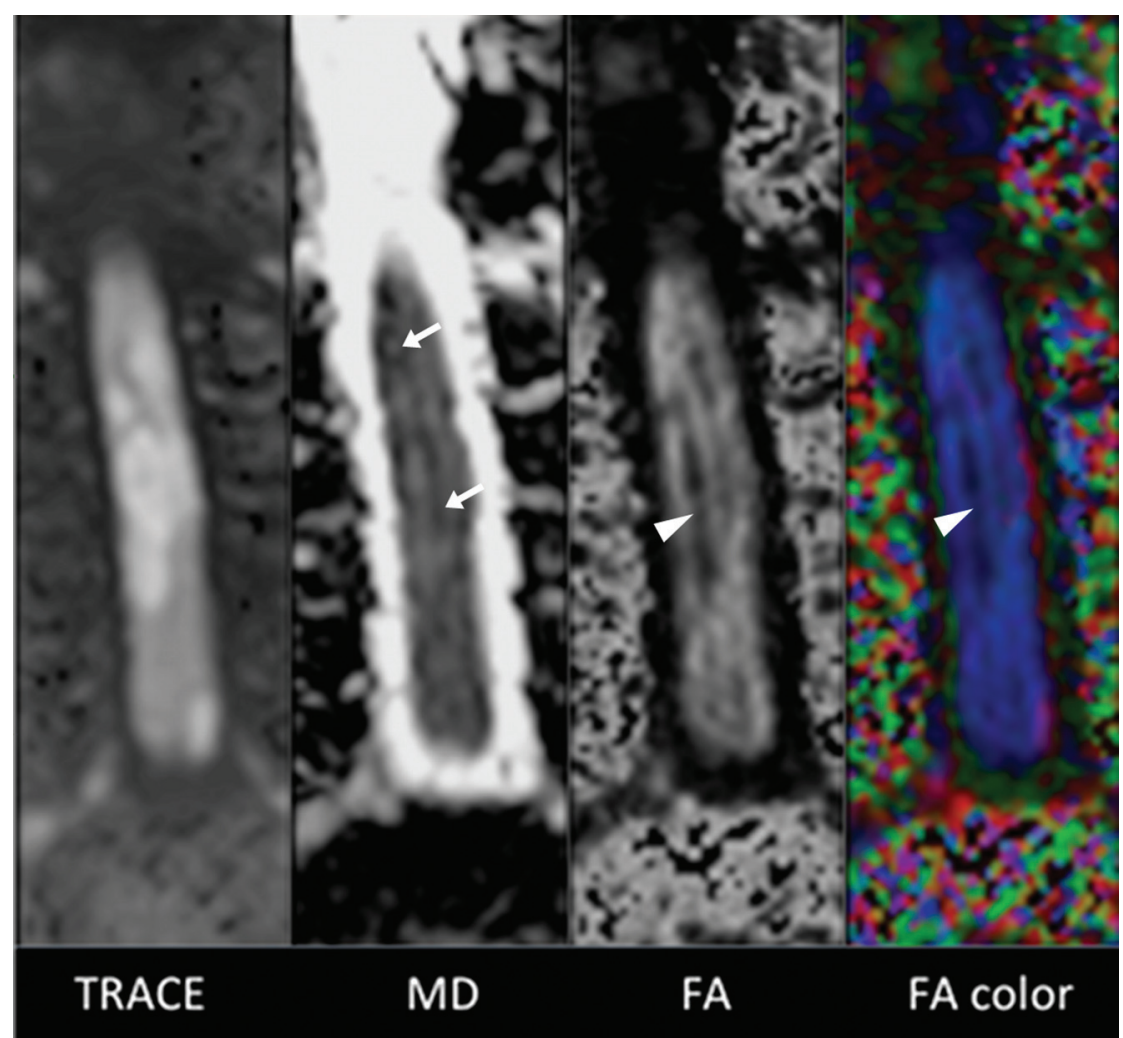

FIG 3. Diffusion tensor images of a patient with active inflammatory lesions of the cervical spinal cord. Inflammatory active lesions appeared bright on the trace image, with the central decrease in FA (arrowheads) and increase in MD. The marked increase in FA and decrease in MD (arrows) observed at the margins of the inflammatory lesions on parametric maps explained the values measured after tractography within the posterior and lateral funiculi for this patient (ie, $\mathrm{FA}=0.65$ and $\mathrm{MD}=0.83 \times 10^{-3} \mathrm{~mm}^{2} / \mathrm{s}$ )

Hyperintensities of the spinal cord represent a broad spectrum of lesions, from reversible to more severe lesions. DTI parameters are more sensitive than T2 signal analysis in assessing patients with myelitis. ${ }^{9}$ We observed lower FA and higher MD values in patients with chronic myelitis, as previously reported. ${ }^{30}$ Decrease in the FA values may reflect the degree of microstructural disorganization of the spinal cord, suggesting either local extracellular edema or a smaller number of fibers matching a larger extracellular space or both. ${ }^{9}$ In addition, radial diffusivity may serve as a marker of overall tissue integrity within chronic MS lesions. ${ }^{8} \mathrm{We}$ also observed an increase in FA associated with a marked decrease in diffusivity values in patients with active lesions, a finding previously suggested in active brain lesions. ${ }^{10}$ Increased FA within gadolinium-enhancing lesions may be related to the resolution of edema and the inflammatory infiltrates. Further studies are required in a larger population of patients with different patterns of myelitis to confirm these results.

Extensive spinal cord lesions are more frequent in neuromyelitis optica than in MS. Neuromyelitis optica and MS are 2 autoimmune demyelinating diseases, but their physiopathology extensively differs. ${ }^{31}$ Thus, this observation could easily explain why statistical differences concerning DTI parameter values were most likely observed when we compared HC with homogeneous subgroups (ie, separating patients with and without acute lesions and patients with and without MS) rather than with our inhomogeneous total cohort. Otherwise correlations between DTI parame- ters and clinical parameters were also most likely observed within the subgroups rather than in our total cohort.

Correlations between clinical and DTI parameters validated our approach. For some of the tested parameters, we observed only trends after applying the Bonferroni correction. By increasing the size of each group, we may find more and better correlations. However, in this study, we observed that both FA and the ratio of $\mathrm{aD}$ and $\mathrm{rD}$ were lower in progressive forms of MS. Indeed, patients with progressive forms of MS were more disabled than those with relapsing-remitting MS: They had longer disease duration, higher Expanded Disability Status Scale scores, and also greater neuronal damage measured by brain and gray matter atrophy. ${ }^{32}$ Patients with primary-progressive MS were also characterized by spinal cord atrophy. ${ }^{33}$ Even if our different groups are small, these findings suggest the validity of our technique.

Our study had some limitations. We used a DTI sequence with a b-value of 600 and 15 directions due to a compromise between image quality and time acquisition. With such parameters, sensitivity to axial or lower diffusion fibers might be decreased and longitudinal fibers will dominate within the tracts obtained. However, this study was focused on patients with myelitis for whom the longitudinal fibers of the spinal cord such as corticospinal or lemniscal funiculi are more frequently involved. Triggering each section may improve the image quality. However, to reduce the scanning time, we performed triggering on each section group with an interleaved acquisition order. Hand-drawn regions of interest were used to define the seed masks. This approach may decrease the reproducibility of the DTI metrics because the number of voxels in the FOV may be small. This was especially challenging in the subjects with myelitis who had cord atrophy. However, to approximate the known locations of the posterior and lateral columns, we drew ROIs on MD images registered with anatomic T2-weighted images.

Given the curvature of the spinal cord, signal differences could be observed at the outer margins of the cord due to partial volume averaging with CSF. However, when we defined seed ROIs, there was a consistent sparing of the outer margin of the cervical cord to minimize this bias. The values we reported were gray and white averaged FA values; however, spinal white matter fibers have a craniocaudal orientation and thus are very anisotropic, contrary to gray matter, which is more isotropic. We did not image the whole spinal cord. Indeed the cervical cord is easier to assess by MR imaging due to its larger size and decreased mobility compared with the thoracic cord. ${ }^{34}$ We have calculated DTI metrics along the entire tract from $\mathrm{C} 1$ to $\mathrm{C} 7$. With such an approach, the 
Table 1: DTI parameters in healthy controls, the total cohort of patients with myelitis, and different subgroups

\begin{tabular}{|c|c|c|c|c|c|c|}
\hline & \multirow[b]{2}{*}{$\begin{array}{l}\text { Healthy } \\
\text { Controls }\end{array}$} & \multirow{2}{*}{$\begin{array}{l}\text { All Patients with } \\
\text { or without } \\
\text { Active Lesions }\end{array}$} & \multicolumn{3}{|c|}{ Patients without Active Lesions } & \multirow[b]{2}{*}{$\begin{array}{l}\text { Patients with } \\
\text { Active Lesions }\end{array}$} \\
\hline & & & $\begin{array}{l}\text { Patients with MS } \\
\text { and Other Patients }\end{array}$ & $\begin{array}{l}\text { Patients } \\
\text { with MS }\end{array}$ & $\begin{array}{l}\text { Other } \\
\text { Patients }^{\mathrm{a}}\end{array}$ & \\
\hline No. & 12 & 40 & 34 & 20 & 14 & 6 \\
\hline $\operatorname{Sex}(F / M)$ & 7:5 & 19:21 & 18:16 & 9:11 & 9:5 & 1:5 \\
\hline Age (yr) (mean) & $31.6 \pm 9.1$ & $43.3 \pm 12.0$ & $43.6 \pm 11.8$ & $40.5 \pm 13.0$ & $48.0 \pm 7.5$ & $41.5 \pm 14.7$ \\
\hline FA (mean) & $0.61 \pm 0.01$ & $0.54 \pm 0.06$ & $0.52 \pm 0.05$ & $0.54 \pm 0.04$ & $0.50 \pm 0.05$ & $0.64 \pm 0.05$ \\
\hline $\operatorname{MD}\left(10^{-3} \mathrm{~mm}^{2} / \mathrm{s}\right)$ (mean) & $1.17 \pm 0.07$ & $1.30 \pm 0.28$ & $1.38 \pm 0.22$ & $1.40 \pm 0.20$ & $1.34 \pm 0.25$ & $0.84 \pm 0.13$ \\
\hline $\mathrm{El}=\mathrm{aD}\left(10^{-3} \mathrm{~mm}^{2} / \mathrm{s}\right)$ (mean) & $1.96 \pm 0.10$ & $2.02 \pm 0.36$ & $2.12 \pm 0.28$ & $2.17 \pm 0.25$ & $2.04 \pm 0.32$ & $1.45 \pm 0.17$ \\
\hline E2 $\left(10^{-3} \mathrm{~mm}^{2} / \mathrm{s}\right)$ (mean) & $0.92 \pm 0.05$ & $1.10 \pm 0.27$ & $1.18 \pm 0.22$ & $1.18 \pm 0.20$ & $1.18 \pm 0.25$ & $0.68 \pm 0.12$ \\
\hline E3 $\left(10^{-3} \mathrm{~mm}^{2} / \mathrm{s}\right)$ (mean) & $0.63 \pm 0.06$ & $0.77 \pm 0.24$ & $0.83 \pm 0.19$ & $0.84 \pm 0.17$ & $0.82 \pm 0.23$ & $0.39 \pm 0.13$ \\
\hline $\mathrm{rD}\left(10^{-3} \mathrm{~mm}^{2} / \mathrm{s}\right)$ (mean) & $0.77 \pm 0.05$ & $0.93 \pm 0.26$ & $1.00 \pm 0.20$ & $1.00 \pm 0.19$ & $1.00 \pm 0.23$ & $0.53 \pm 0.12$ \\
\hline Ratiob (mean) & $2.5 \pm 0.08$ & $2.25 \pm 0.37$ & $2.15 \pm 0.25$ & $2.20 \pm 0.26$ & $2.09 \pm 0.22$ & $2.82 \pm 0.45$ \\
\hline
\end{tabular}

a Patients with myelitis with other causes of inflammatory myelopathy (neuromyelitis optica, sarcoidosis, Gougerot-Sjögren syndrome, idiopathic).

${ }^{\mathrm{b}}$ Ratios of $\mathrm{aD}$ and $\mathrm{rD}$.

Table 2: Comparison of DTI parameter values among HC, the total cohort of patients (including MS and other cause of inflammatory myelopathy), and different subgroups of patients

\begin{tabular}{lccccc}
\hline & HC vs Total Cohort & HC vs (MS + Other) & HC vs MS & HC vs Other & HC vs Patients with \\
\cline { 3 - 5 } Acute Cervical Lesions \\
\hline FA $\left(10^{-3} \mathrm{~mm}^{2} / \mathrm{s}\right)$ & $P=.001^{\mathrm{a}, \mathrm{b}}$ & $P<.0001^{\mathrm{a}, \mathrm{b}}$ & $P<.0001^{\mathrm{a}, \mathrm{b}}$ & $P<.0001^{\mathrm{a}, \mathrm{b}}$ & $P=0.091$ \\
$\mathrm{MD}\left(10^{-3} \mathrm{~mm}^{2} / \mathrm{s}\right)$ & $P=.131$ & $P=.002^{\mathrm{a}}$ & $P<.0001^{\mathrm{a}, \mathrm{b}}$ & $P=.025^{\mathrm{a}}$ & $P<.0001^{\mathrm{a}, \mathrm{b}}$ \\
$\mathrm{El}\left(10^{-3} \mathrm{~mm}^{2} / \mathrm{s}\right)$ & $P=.610$ & $P=.075$ & $P=.011^{\mathrm{a}}$ & $P=.432$ & $P<.0001^{\mathrm{a}, \mathrm{b}}$ \\
$\mathrm{E2}\left(10^{-3} \mathrm{~mm}^{2} / \mathrm{s}\right)$ & $P=.014^{\mathrm{a}}$ & $P<.0001^{\mathrm{a}, \mathrm{b}}$ & $P<.0001^{\mathrm{a}, \mathrm{b}}$ & $P=.005^{\mathrm{a}}$ & $P<.0001^{\mathrm{a}, \mathrm{b}}$ \\
E3 $\left(10^{-3} \mathrm{~mm}^{2} / \mathrm{s}\right)$ & $P=.056$ & $P<.0001^{\mathrm{a}, \mathrm{b}}$ & $P<.0001^{\mathrm{a}, \mathrm{b}}$ & $P=.012^{\mathrm{a}}$ & $P<.0001^{\mathrm{a}, \mathrm{b}}$ \\
rD $\left(10^{-3} \mathrm{~mm}^{2} / \mathrm{s}\right)$ & $P=.040^{\mathrm{a}}$ & $P<.0001^{\mathrm{a}, \mathrm{b}}$ & $P<.0001^{\mathrm{a}, \mathrm{b}}$ & $P=.003^{\mathrm{a}}$ & $P<.0001^{\mathrm{a}, \mathrm{b}}$ \\
Ratio & $P<.0001^{\mathrm{a}, \mathrm{b}}$ & $P<.0001^{\mathrm{a}, \mathrm{b}}$ & $P<.0001^{\mathrm{a}, \mathrm{b}}$ & $P=.005^{\mathrm{a}}$ & $P=.291$ \\
\hline
\end{tabular}

a Significant $P$ values.

b $P$ values remaining significant $(P<.05)$ after Bonferroni correction.

Table 3: Clinical and DTI parameters of patients with MS without active lesions

\begin{tabular}{lcccc}
\hline & RRMS $(\boldsymbol{n}=12)$ & PPMS $(\boldsymbol{n}=\mathbf{4})$ & SPMS $(\boldsymbol{n}=\mathbf{4})$ & Progressive MS $(\boldsymbol{n}=\mathbf{8})$ \\
\hline EDSS (median [min;max]) & $3.0[0 ; 8]$ & $6.75[4 ; 8]$ & $6.5[6.5 ; 7.5]$ & $6.5[4 ; 8]$ \\
Pyramidal FS (median [min;max]) & $2[0 ; 5]$ & $4[2 ; 5]$ & $4[4 ; 4]$ & $4[2 ; 5]$ \\
Sensory FS (median [min;max]) & $2[0 ; 3]$ & $3[1 ; 4]$ & $3.5[3 ; 4]$ & $3[1 ; 4]$ \\
FA (mean) & $0.56 \pm 0.03$ & $0.53 \pm 0.04$ & $0.49 \pm 0.03$ & $0.51 \pm 0.04$ \\
MD $\left(10^{-3} \mathrm{~mm}^{2} / \mathrm{s}\right)$ (mean) & $1.36 \pm 0.15$ & $1.40 \pm 0.26$ & $1.52 \pm 0.25$ & $1.46 \pm 0.25$ \\
Ratio (mean) & $2.29 \pm 0.26$ & $2.13 \pm 0.20$ & $1.99 \pm 0.18$ & $2.06 \pm 0.19$ \\
\hline
\end{tabular}

Note:-min indicates minimum; max, maximum; FS, functional score; RRMS, relapsing-remitting MS; SPMS, secondary-progressive MS; PPMS, primary-progressive MS; EDSS, Expanded Disability Status Scale.

a $0-6$.

${ }^{\mathrm{b}}$ Ratios of $\mathrm{aD}$ and $\mathrm{rD}$

measure of DTI indices was potentially less sensitive in cases of limited lesions of the spinal cord due to the averaged values. However, despite this shortcoming, findings showed that all patients with myelitis could be distinguished from healthy volunteers. Furthermore, histogram analysis of DTI indices may be provided to improve the sensitivity of the technique.

\section{CONCLUSIONS}

We showed that the pulse-triggered single-shot EPI DTI sequence with the rFOV technique was reliable for the routine assessment of quantitative DTI indices over the cervical spinal cord at 3T. The further finding that DTI indices were associated with disability scores suggested the clinical value of this approach for the management of patients with myelitis.

Disclosures: Olivier Outteryck—UNRELATED: Payment for Lectures (including service on Speakers Bureaus): Biogen-Idec, Bayer, Sanofi, Travel/Accommodations/ Meeting Expenses Unrelated to Activities Listed: Bayer, Biogen-Idec, TEVA, Sanofi, Novartis, Merck-Serono. Hélène Zéphir-UNRELATED: Board Membership: BiogenIdec, Sanofi, Consultancy: Biogen-Idec, TEVA, Sanofi, Merck, Novartis, Bayer, Payment for Lectures (including service on Speakers Bureaus): Biogen-Idec, ${ }^{*}$ Bayer, ${ }^{*}$
Novartis, ${ }^{*}$ Travel/Accommodations/Meeting Expenses Unrelated to Activities Listed: Biogen-Idec, * Merck, ${ }^{*}$ Novartis, ${ }^{\star}$ Bayer, * Sanofi, ${ }^{*}$ Teva. * David Chéchin—UNRELATED: Employment: Philips Healthcare, Comments: I am employed by Philips Healthcare, and this work was done on a Philips 3T MRI system. I supported this work by installing, evaluating, and optimizing the sequences. Patrick Vermersch-UNRELATED: Board Membership: honoraria from Biogen-Idec, TEVA, Sanofi, Bayer, Novartis, Almirall, Consultancy: honoraria from Biogenidec, TEVA, Sanofi, Bayer, Novartis, GlaxoSmithKline, Payment for Development of Education Presentations: from GlaxoSmithKline, Almirall, Travel/Accommodations/Meeting Expenses Unrelated to Activities Listed: travel expenses from Biogen-Idec, TEVA, Sanofi, Bayer, Novartis. *Money paid to the institution.

\section{REFERENCES}

1. Budzik JF, Balbi V, Le Thuc V, et al. Diffusion tensor imaging and fibre tracking in cervical spondylotic myelopathy. Eur Radiol 2011;21:426-33

2. Ellingson BM, Ulmer JL, Kurpad SN, et al. Diffusion tensor MR imaging of the neurologically intact human spinal cord. AJNR Am J Neuroradiol 2008;29:1279-84

3. Ellingson BM, Ulmer JL, Kurpad SN, et al. Diffusion tensor MR imaging in chronic spinal cord injury. AJNR Am J Neuroradiol 2008;29:1976-82

4. Mohamed FB, Hunter LN, Barakat N, et al. Diffusion tensor imaging 
of the pediatric spinal cord at 1.5T: preliminary results. AJNR Am J Neuroradiol 2011;32:339-45

5. Shanmuganathan K, Gullapalli RP, Zhuo J, et al. Diffusion tensor MR imaging in cervical spine trauma. AJNR Am J Neuroradiol 2008;29:655-59

6. Freund $\mathrm{P}$, Wheeler-Kingshott $\mathrm{C}$, Jackson J, et al. Recovery after spinal cord relapse in multiple sclerosis is predicted by radial diffusivity. Mult Scler 2010;16:1193-202

7. Hesseltine SM, Law M, Babb J, et al. Diffusion tensor imaging in multiple sclerosis: assessment of regional differences in the axial plane within normal-appearing cervical spinal cord. AJNR Am J Neuroradiol 2006;27:1189-93

8. Klawiter EC, Schmidt RE, Trinkaus K, et al. Radial diffusivity predicts demyelination in ex vivo multiple sclerosis spinal cords. Neuroimage 2011;55:1454-60

9. Renoux J, Facon D, Fillard P, et al. MR diffusion tensor imaging and fiber tracking in inflammatory diseases of the spinal cord. AJNR Am J Neuroradiol 2006;27:1947-51

10. Fox RJ, Cronin T, Lin J, et al. Measuring myelin repair and axonal loss with diffusion tensor imaging. AJNR Am J Neuroradiol 2011;32:85-91

11. Farzaneh F, Riederer SJ, Pelc NJ. Analysis of T2 limitations and offresonance effects on spatial resolution and artifacts in echo-planar imaging. Magn Reson Med 1990;14:123-39

12. Bammer R, Augustin M, Prokesch RW, et al. Diffusion-weighted imaging of the spinal cord: interleaved echo-planar imaging is superior to fast spin-echo. J Magn Reson Imaging 2002;15:364-73

13. Melhem ER. Technical challenges in MR imaging of the cervical spine and cord. Magn Reson Imaging Clin N Am 2000;8:435-52

14. Wilm BJ, Gamper U, Henning A, et al. Diffusion-weighted imaging of the entire spinal cord. NMR Biomed 2009;22:174-81

15. Wilm BJ, Svensson J, Henning A, et al. Reduced field-of-view MRI using outer volume suppression for spinal cord diffusion imaging. Magn Reson Med 2007;57:625-30

16. Kurtzke JF. Rating neurologic impairment in multiple sclerosis: an expanded disability status scale (EDSS). Neurology 1983;33:1444-52

17. Zaharchuk G, Saritas EU, Andre JB, et al. Reduced field-of-view diffusion imaging of the human spinal cord: comparison with conventional single-shot echo-planar imaging. AJNR Am J Neuroradiol 2011;32:813-20

18. Smith SM, Jenkinson M, Woolrich MW, et al. Advances in functional and structural MR image analysis and implementation as FSL. Neuroimage 2004;23(suppl 1):S208-19

19. Woolrich MW, Jbabdi S, Patenaude B, et al. Bayesian analysis of neuroimaging data in FSL. Neuroimage 2009;45:S173-86

20. Saritas EU, Cunningham $\mathrm{CH}$, Lee JH, et al. DWI of the spinal cord with reduced FOV single-shot EPI. Magn Reson Med 2008;60:468-73

21. Habib J, Auer DP, Morgan PS. A quantitative analysis of the benefits of cardiac gating in practical diffusion tensor imaging of the brain. Magn Reson Med 2010;63:1098-103

22. Summers P, Staempfli P, Jaermann T, et al. A preliminary study of the effects of trigger timing on diffusion tensor imaging of the human spinal cord. AJNR Am J Neuroradiol 2006;27:1952-61

23. Demir A, Ries M, Moonen CT, et al. Diffusion-weighted MR imaging with apparent diffusion coefficient and apparent diffusion tensor maps in cervical spondylotic myelopathy. Radiology 2003;229:37-43

24. Van Hecke W, Leemans A, Sijbers J, et al. A tracking-based diffusion tensor imaging segmentation method for the detection of diffusion-related changes of the cervical spinal cord with aging. J Magn Reson Imaging 2008;27:978-91

25. Agosta F, Benedetti B, Rocca MA, et al. Quantification of cervical cord pathology in primary progressive MS using diffusion tensor MRI. Neurology 2005;64:631-35

26. Benedetti B, Valsasina P, Judica E, et al. Grading cervical cord damage in neuromyelitis optica and MS by diffusion tensor MRI. Neurology 2006;67:161-63

27. Clark CA, Werring DJ, Miller DH. Diffusion imaging of the spinal cord in vivo: estimation of the principal diffusivities and application to multiple sclerosis. Magn Reson Med 2000;43:133-38

28. Ohgiya Y, Oka M, Hiwatashi A, et al. Diffusion tensor MR imaging of the cervical spinal cord in patients with multiple sclerosis. Eur Radiol 2007;17:2499-504

29. Valsasina P, Rocca MA, Agosta F, et al. Mean diffusivity and fractional anisotropy histogram analysis of the cervical cord in MS patients. Neuroimage 2005;26:822-28

30. van Hecke W, Nagels G, Emonds G, et al. A diffusion tensor imaging group study of the spinal cord in multiple sclerosis patients with and without T2 spinal cord lesions. J Magn Reson Imaging 2009;30:25-34

31. Popescu BF, Lucchinetti CF. Pathology of demyelinating diseases. Annu Rev Pathol 2012;7:185-217

32. Fisher E, Lee J, Nakamura K, et al. Gray matter atrophy in multiple sclerosis: a longitudinal study. Ann Neurol 2008;64:255-65

33. Bieniek M, Altmann D, Davies G, et al. Cord atrophy separates early primary progressive and relapsing remitting multiple sclerosis. J Neurol Neurosurg Psychiatry 2006;77:1036-39

34. Stankiewicz JM, Neema M, Alsop DC, et al. Spinal cord lesions and clinical status in multiple sclerosis: a 1.5 T and $3 \mathrm{~T}$ MRI study. J Neurol Sci 2009;279:99-105 